\title{
La planificación centrada en la persona y su pertinencia práctica en los servicios sociales: opinión de los profesionales
}

\section{Eva Benito Herráez}

Facultad de Psicología, Universidad Nacional de Educación a Distancia (UNED) <evabenito@pip.udl.cat>

\section{Carles Alsinet Mora}

Departament de Pedagogia i Psicologia, Universitat de Lleida

\section{Araceli Maciá Antón}

Departamento de Metodología de las Ciencias del Comportamiento, Universidad Nacional de Educación a Distancia (UNED)

Pertsonan zentratutako planifikazioa da bizikalitatea eta ongizate pertsonala sustatu nahi duen eredu bat eskuartze pertsonalizatu eta pertsonaren premia, gaitasun eta potentzialtasunetara egokituz. Planifikazio tradizionaletik pertsonan zentratutako planifikaziora igarotzeko, beharrezkoa da aldaketa bat gertatzea eskuartze profesionalean, eta berau lortzen da prestakuntza eta sentsibilizazioaren bidetik. Ikerketa honetan analizatzen dira ereduak eskaintzen dituen aukera, muga eta abantailak gizarte-zerbitzuetako profesionalen talde baten eskutik. Lortutako emaitzek erakusten dute profesional horiek garatzen duten eskuartze horren metodologiaren egokitasuna.

\section{GAKO-HITZAK:}

Pertsonan zentratutako planifikazioa, gizartezerbitzuak, profesionalak, ebaluazioa.
La planificación centrada en la persona es un modelo que pretende promover la calidad de vida y el bienestar personal mediante una intervención personalizada y adaptada a las necesidades, capacidades y potencialidades de la persona. El tránsito de la planificación tradicional a la planificación centrada en la persona requiere un cambio en la intervención profesional que se consigue mediante la formación y la sensibilización. El presente estudio analiza las valoraciones de un grupo de profesionales de servicios sociales sobre las posibilidades del modelo, sus limitaciones y ventajas. Los resultados obtenidos ponen de manifiesto la pertinencia de la metodología en la intervención que desarrollan los profesionales.

\section{Palabras Clave:}

Planificación centrada en la persona, servicios sociales, profesionales, evaluación. 


\section{Introducción}

Los servicios sociales se encuentran en un momento de cambio, de replanteamiento de su función, y especialmente de transformación hacia un modelo que priorice la atención personalizada adaptada a las necesidades, capacidades y potencialidades de la persona, que tenga en cuenta el contexto en el que vive, y promueva la calidad de vida y el bienestar personal (Duque, 2012). Para conseguir el tránsito hacia este modelo centrado en la persona, individualizado y basado en las capacidades y en el fomento de la calidad de vida, es precisa una brújula teórica y metodológica. En este sentido, la planificación centrada en la persona se está convirtiendo en un paradigma de intervención social (Fantova, 2004).

El presente estudio pretende analizar las valoraciones que un grupo de profesionales que desarrollan su labor en los servicios sociales realizan acerca de las posibilidades de esta metodología, sus limitaciones y ventajas, para, posteriormente, incorporar los resultados obtenidos al proceso de diseño de un proyecto piloto de atención a personas en situación de sin hogar basado en este paradigma.

En las siguientes líneas, se define brevemente qué es la planificación centrada en la persona y sus posibilidades de aplicación y desarrollo en los servicios sociales, así como las limitaciones y dificultades que supone su implementación, especialmente en lo relativo a la formación y sensibilización de los profesionales. En el resto del artículo, se expondrán los resultados obtenidos de la investigación y se discutirán sus implicaciones prácticas.

\section{La planificación centrada en la persona como alternativa metodológica en los servicios sociales}

La planficiación centrada en la persona fue iniciada en el ámbito de la discapacidad intelectual en Estados Unidos en los años ochenta por O’Brien, Mount, Pearpoint, Forest y Smull (Mount y Zwernik, 1988; Pearpoint, O’Brien y Forest, 1993; Smull, 1998), y ofrece una forma de comprender las experiencias de las personas con discapacidad intelectual utilizando los apoyos y redes sociales para mejorar su calidad de vida. Se fundamenta en los derechos de las personas con discapacidad e incorpora los principios de independencia, opción e inclusión. Es una metodología que se basa en la creencia de que todos los seres humanos somos diferentes y se debe respetar nuestra individualidad.

Según Holburn (2003), es una mezcla de ideología y estrategia, ya que, por un lado, defiende la individualización y la autodeterminación como principios centrales de los servicios de apoyo a las personas, y por otro, facilita que la persona identifique las metas que quiere lograr en su vida, planificando los pasos a seguir y utilizando métodos específicos para producir cambios en las vidas de las personas.

La planificación centrada en la persona adopta el modelo de calidad de vida propuesto por Schalock y Verdugo (2003). Los autores consideran la calidad de vida como un fenómeno subjetivo, basado en la percepción que tiene una persona de varios aspectos de las experiencias de su vida, incluyendo las características personales, las condiciones objetivas de vida y las percepciones de los otros significativos. Se entiende como un concepto multidimensional, compuesto por ocho dimensiones: el bienestar físico, el bienestar emocional, las relaciones interpersonales, la inclusión social, el desarrollo personal, el bienestar material, la autodeterminación y los derechos.

Según Schalock (1996), pese a no ser un constructo nuevo, lo que es innovador es su utilización como principio organizador de servicios de apoyo para lograr que las personas con dificultades o en situación de exclusión mejoren su vida. Para Schalock y Verdugo (2009), el concepto de calidad de vida provoca pensar diferente sobre las personas que se encuentran al margen de la sociedad y promover un cambio en las organizaciones, en los sistemas y en la comunidad, con el objetivo de incrementar su bienestar personal y reducir la exclusión. A día de hoy, es considerado un elemento imprescindible para el desarrollo de políticas y programas de inclusión social dirigidos a colectivos en riesgo de exclusión. Según los mismos autores, ello es debido a que se concibe como: a) una noción sensibilizadora que proporciona referencia y guía desde la perspectiva del individuo, centrada en las dimensiones centrales de una vida de calidad; b) un constructo social que guía estrategias de intervención; y c) un marco conceptual para evaluar resultados de calidad y un criterio para evaluar la eficacia de dichas estrategias (Schalock y Verdugo, 2003, 2009).

La implementación de proyectos basados en la planificación centrada en la persona se produce fundamentalmente en países anglosajones y tienen como objetivo trabajar con las personas, las organizaciones y las comunidades utilizando un modelo centrado en la persona, en la mayoría de los casos en torno a personas con discapacidad. Sin embargo, Neill et al. (2009) plantean que se debe ampliar a cualquier recurso o servicio que tenga por objetivo el apoyo y acompañamiento a las personas, familias o comunidades. En la misma línea, Fernández Muñoz (2011) postula que la metodología supone un cambio de paradigma en la intervención en servicios sociales, al reenfocarse éstos hacia la autonomía personal y la personalización. Para el autor, se basa en las capacidades y potencialidades personales, y no en las dificultades, y no se sirve de la mera prestación de servicios, sino del reconocimiento y del ejercicio pleno de los derechos, de las oportunidades y de la responsabilidad ciudadana, así como de la práctica de relaciones 
simétricas y de igualdad, que tengan en cuenta a la persona, su entorno, y las relaciones y los mapas personales. Éste es uno de los principales motivos por los cuales la metodología es considerada como una oportunidad de avance en la intervención social que se desarrolla en los servicios sociales y comunitarios.

\section{La práctica de la planificación centrada en la persona en el ámbito de servicios sociales}

A pesar de haber nacido en el ámbito de la discapacidad, las experiencias en España en torno a la extensión de la metodología hacia colectivos relacionados con la inclusión y los servicios sociales empiezan a cobrar importancia, aunque no son muy numerosas. Uno de los ejemplos más evidentes es el desarrollo de la escala Gencat de calidad de vida (Verdugo, 2008), elaborada por el Instituto Universitario de Integración en la Comunidad (Inico) de la Universidad de Salamanca por encargo de la Generalitat de Catalunya y enmarcada en el Plan de Mejora de la Calidad del Instituto Catalán de Asistencia y Servicios Sociales (ICASS). La escala es un instrumento de evaluación objetiva basado en el modelo multidimensional de calidad de vida de Schalock y Verdugo (2003), y su finalidad principal es ser utilizada para la mejora continua de los servicios sociales, al tener en cuenta las necesidades y preferencias de las personas en el diseño de los planes de atención y soporte personal.

La herramienta se está utilizando para definir la intervención social en colectivos en situación de vulnerabilidad y riesgo social, como por ejemplo, en el estudio de Arias Martínez et al. (2010), que analiza la calidad de vida en personas con drogodependencias. Actuaciones más recientes muestran que, pese a no estar específicamente diseñada para medir la calidad de vida de personas sin hogar, empiezan a aparecer tímidamente experiencias de aplicación de la escala a este colectivo, siendo otro ejemplo más de la extensión del paradigma en el ámbito de los servicios sociales. Entre las más destacables, se encuentra la desarrollada por Verdugo et al. (2010), quienes analizan la valoración objetiva de la calidad de vida de trabajadores con discapacidad o en situación de exclusión social participantes en un programa nacional de empleo con apoyo. La pertinencia de la escala, así como sus posibilidades de aplicación e idoneidad para medir la calidad de vida del colectivo de personas sin hogar se hacen evidentes en estudios como el de Aguirre (2013), que utiliza los resultados obtenidos para planificar intervenciones específicas y adaptadas a las necesidades de los usuarios de servicios sociales destinados a este grupo de población, o el de Benito, Alsinet y Maciá (2015), que analiza las diferencias en el nivel de calidad de vida entre personas sin hogar con y sin discapacidad.

\section{Ventajas e inconvenientes de la implementación de la metodología}

Como hemos comentado anteriormente, para Schalock (1995), la planificación centrada en la persona supone un nuevo paradigma en la intervención con personas con discapacidad que está fundamentado en tres elementos de cambio: a) de un sistema centrado en las limitaciones de la persona a otro centrado en el contexto y la interacción bajo los supuestos del enfoque ecológico de Bronfenbrenner (1979); b) de un modelo que se desarrolla a partir de la eficacia de los servicios, programas y actividades, a otro centrado en los avances en la calidad de vida y las mejoras personales; y c) de un sistema centrado en los profesionales a otro donde la persona es el centro de la acción social, con el acompañamiento de sus grupos sociales de referencia y su comunidad.

La metodología quiere romper con el modelo de planificación más clásico o tradicional que parte de las dificultades del individuo, no de sus capacidades, y en el cual, la persona no es el centro del proceso, sino que lo es el profesional. Un cambio siempre es complicado, especialmente si lo que se modifica es una forma concreta de pensar y de actuar. Si además la transformación se produce en organizaciones, pueden suponer un gran riesgo y plantear nuevas necesidades. Las resistencias al cambio, por parte de los líderes, los equipos y los profesionales suelen ser el principal inconveniente del cambio y es preciso aceptarlas como un hecho natural y un elemento esencial del proceso de transformación (Coghlam, 1993).

Sin embargo, para Martínez Rodríguez (2013) existen elementos que pueden actuar como facilitadores, y algunos de esos factores que pueden favorecer el éxito en la implementación de la planificación son el liderazgo claro de los responsables de la organización, el compromiso de los profesionales, la progresividad en su desarrollo, y la formación y acompañamiento de los profesionales en todo el proceso.

Martínez Rodríguez et al. (2014) consideran que las resistencias al cambio en la forma de pensar y de actuar que se producen entre los profesionales al aceptar la metodología pueden superarse si se utilizan estrategias como la identificación de resistencias, la empatía o la personalización de recursos propios, del equipo de trabajo y de las demás personas participantes del proceso. Consideran que la promoción de acciones de formación e intercambio profesional es un elemento importante a la hora de aumentar y mejorar las competencias que capacitan a los profesionales para hacer frente a los cambios metodológicos. Trabajos como el de Duque (2012) o el de Edwarsson, Sandman y Borell (2014) apoyan esta premisa. El primero de ellos afirma que, para que se produzca el cambio de mentalidad, es preciso un proceso de formación continua que fomente la reflexión y la adquisición de las competencias 
necesarias para que los implicados en la labor profesional tengan presentes los principios y premisas de la intervención en calidad de vida y autonomía que representa la planificación centrada en la persona. En el segundo, se otorga especial importancia a la transferencia del conocimiento adquirido por los profesionales en el proceso de formación, y a la práctica diaria como elemento clave del éxito de la implementación de la metodología.

La sensibilización y la formación son, entonces, fundamentales a la hora de implementar el paradigma en una organización. De hecho, para Martínez Rodríguez et al. (2014) suponen la primera de las fases que proponen para aplicar un modelo de atención centrado en la persona en una organización. La sensibilización y el logro de un liderazgo de cambio compartido e interno son los dos primeros pasos para conseguir el cambio metodológico, e implican la formación de los profesionales, $y$, si es necesario, también de las personas destinatarias, de los grupos de apoyo o de las familias. Boersma et al. (2014), tras realizar una revisión de la literatura relativa a la atención e intervención dirigida a personas con demencia, concluyeron que es preciso el uso de múltiples estrategias de implementación fundamentadas en la formación si se quiere garantizar el éxito en la intervención, y que ésta debe desarrollarse a largo plazo, no únicamente en las fases iniciales.

Apoyándose en estas afirmaciones, el presente trabajo analiza las opiniones y valoraciones de los profesionales de servicios sociales de la ciudad de Lleida acerca de la planificación centrada en la persona, especialmente a lo relativo a las dificultades y potencialidades en el momento de adaptar la metodología y sus principios de intervención a los destinatarios. Para ello, y después de un curso formativo, se recogió información relativa a la aplicabilidad y facilidad de adaptación, así como a las dificultades y ventajas que puede aportar la metodología a la práctica diaria de los profesionales encuestados. Los resultados obtenidos han resultado de interés para el diseño de un proyecto de adaptación del modelo destinado a personas sin hogar, que se encuentra actualmente en fase de implementación.

\section{Metodología}

\subsection{Diseño y procedimiento}

Para analizar las opiniones de los profesionales, se ha realizado un estudio transversal descriptivo basado en una encuesta. El objetivo principal ha sido conocer sus opiniones acerca de la complejidad, aplicabilidad y facilidad de adaptación de la metodología en el ámbito de los servicios sociales. También se ha recogido información acerca de las dificultades y ventajas que puede aportar a la práctica diaria.

Para alcanzar el objetivo, se procedió a convocar a los profesionales que conforman los equipos de servicios sociales a una sesión de formación en la planificación centrada en la persona diseñada específicamente para la ocasión. Esta sesión incluyó una serie de contenidos relacionados con los conceptos básicos, principios y enfoques, así como la presentación de diferentes experiencias de implementación de la metodología en el ámbito de los servicios sociales. Tras la sesión, se solicitó la participación voluntaria en el estudio mediante la cumplimentación de la encuesta de valoración diseñada también ad hoc. Una vez recogida la información, se procedió al análisis de los datos y a su posterior comunicación a los participantes.

\subsection{Participantes}

La población sobre la cual se ha diseñado el estudio ha sido los y las profesionales de atención social $^{1}$ (trabajadores sociales, educadores sociales y trabajadores familiares) que forman parte de los equipos de servicios sociales de atención primaria de la ciudad de Lleida, de la que se ha extraído una muestra de 36 participantes. El $44 \%$ (16 profesionales) corresponde a profesionales del trabajo social, mientras que el $42 \%$ son educadores sociales (15 participantes). Dos profesionales (el $5 \%$ de la muestra) ejercen como trabajadores familiares, y han participado también 3 jefas de servicio (que suponen el $8 \%$ restante). Respecto al lugar de trabajo, la mayoría desempeñan sus funciones en los servicios sociales de atención primaria (el 69\% del total), mientras que aquellos que lo hacen en la oficina de atención a personas sin hogar y en la de atención a la dependencia completan el resto de la muestra ( $17 \%$ y $11 \%$ del total, respectivamente).

\subsection{Instrumento de recogida de datos}

El cuestionario utilizado para conocer la opinión de los participantes se ha dividido en tres partes. En la primera, se recoge información básica acerca de la categoría profesional, así como del lugar de trabajo, con el objetivo de analizar los resultados en función de estas dos variables. Tanto la segunda como la tercera parte del cuestionario pretenden obtener las valoraciones de las personas que han participado en el estudio acerca de la metodología: concretamente, sus opiniones acerca de la complejidad, aplicabilidad y facilidad de la metodología, por un lado; y su parecer sobre las ventajas e inconvenientes de su posible implementación en los servicios sociales, por otro.

En el segundo y tercer bloque del cuestionario, las preguntas han sido elaboradas en formato cerrado, pero se han diferenciado las opciones de

${ }^{1}$ Pese a que se ha utilizado terminología neutra siempre que ha sido posible, en el texto aparecen referencias masculinas que en ningún caso pretenden mostrar diferencias o discriminación ninguna, teniendo como único objetivo el de evitar repeticiones que dificultarían la lectura del artículo. Entendemos que ambos sexos están representados en todos y cada uno de los términos empleados. 
respuesta para cada una de ellas. En el segundo bloque, las opciones han oscilado entre la poca, media o alta complejidad, aplicabilidad o facilidad respectivamente, siendo respuestas mutuamente excluyentes. En el tercero, los y las profesionales se han encontrado con respuestas de elección múltiple acerca de las ventajas e inconvenientes que la metodología puede aportar a la práctica cotidiana. Para elaborar las preguntas y las opciones de respuesta de estos dos bloques, se ha partido de los trabajos acerca de las limitaciones y barreras de la planificación centrada en la persona, así como de los relativos a las dificultades en su implementación (Coyle y Moloney, 1999; Pallisera, 2011), orientando el cuestionario según los objetivos del presente estudio.

\subsection{Análisis de datos}

Para el análisis de los resultados obtenidos mediante la aplicación de la encuesta, se ha empleado el programa informático Statistical Package for the Social Sciences (SPSS) 16. Dado el carácter del estudio, los datos ofrecidos son meramente descriptivos y basados en porcentajes.

\section{Resultados}

Seguidamente se presentan los principales resultados del estudio relativos a la valoración de los profesionales sobre la planificación centrada en la persona, así como a las ventajas e inconvenientes de la metodología.

\subsection{Complejidad, aplicabilidad y facilidad de implementación de la metodología}

Como se puede comprobar en la Tabla 1, cerca del $64 \%$ de los profesionales valoran la metodología como poco compleja, frente al $16,7 \%$ que consideran alto su grado de complejidad. Si se analizan los resultados según el grupo profesional, son los trabajadores sociales quienes consideran la metodología menos compleja, aunque con resultados no tan lejanos a los de los educadores sociales. Según el lugar de trabajo, existe bastante dispersión en las respuestas. Si en los servicios sociales de atención primaria seis de cada diez profesionales consideran la metodología como no compleja, en dependencia/asistencia domiciliaria el porcentaje aumenta sensiblemente. Es entre los profesionales de atención a personas sin hogar donde se produce mayor diferencia entre valoraciones: mientras que la mitad de ellos/as valora la metodología como no compleja, un considerable $16 \%$ piensa que sí lo es.

Prácticamente el $75 \%$ de los profesionales consideran que la metodología es aplicable en el ámbito profesional; sin embargo, cabe destacar que un $8,6 \%$ de respuestas manifiestan que no es aplicable y que casi dos de cada diez profesionales no tienen clara su aplicabilidad directa. Si se analizan las respuestas por profesionales, los resultados muestran gran variabilidad. Si ocho de cada diez profesionales de la educación social consideran que el modelo es aplicable a la práctica diaria, el porcentaje se reduce notoriamente para profesionales del trabajo social, que, por consiguiente, consideran en mayor medida que no es aplicable.

Mientras que todos los profesionales de atención a personas sin hogar tienen muy clara la opinión sobre la aplicabilidad metodológica, entre el resto de servicios las valoraciones son más dispersas. Para los profesionales de servicios sociales de atención primaria, las respuestas se reparten entre las tres categorías; pese a ello cerca del $67 \%$ considera que la metodología es aplicable. En dependencia/asistencia domiciliaria, tres cuartos de los profesionales valoran su aplicabilidad.

Tabla 1. Opinión de los profesionales sobre la complejidad, aplicabilidad y facilidad de la planificación centrada en la persona (\%)

\begin{tabular}{|c|c|c|c|c|c|c|c|c|c|}
\hline & \multicolumn{3}{|c|}{ Complejidad } & \multicolumn{3}{|c|}{ Aplicabilidad } & \multicolumn{3}{|c|}{ Facilidad } \\
\hline & $\begin{array}{c}\text { Poco } \\
\text { compleja }\end{array}$ & Media & $\begin{array}{c}\text { Muy } \\
\text { compleja }\end{array}$ & $\begin{array}{c}\text { Poco } \\
\text { aplicable }\end{array}$ & Media & $\begin{array}{c}\text { Muy } \\
\text { aplicable }\end{array}$ & Muy difícil & Media & Muy facil \\
\hline Total & 63,9 & 19,4 & 16,7 & 8,6 & 17,1 & 74,3 & 5,6 & 19,4 & 75,0 \\
\hline Trabajador social & 68,8 & 12,5 & 18,8 & 13,3 & 26,7 & 60,0 & 6,3 & 18,8 & 75,1 \\
\hline Educador social & 60,0 & 26,7 & 13,4 & 6,7 & 6,7 & 86,7 & 6,7 & 13,3 & 80,0 \\
\hline $\begin{array}{l}\text { Trabajador } \\
\text { familiar }\end{array}$ & 50,0 & 0,0 & 50,0 & 0,0 & 100,0 & 0,0 & 0,0 & 50,0 & 50,0 \\
\hline Servicios sociales & 64,0 & 20,0 & 16,0 & 12,5 & 20,8 & 66,6 & 8,0 & 16,0 & 76,0 \\
\hline Dependencia & 75,0 & 0,0 & 25,0 & 0,0 & 25,0 & 75,0 & 0,0 & 50,0 & 50,0 \\
\hline $\begin{array}{l}\text { Atención a } \\
\text { personas sin } \\
\text { hogar }\end{array}$ & 50,0 & 33,3 & 16,7 & 0,0 & 0,0 & 100,0 & 0,0 & 16,7 & 85,3 \\
\hline
\end{tabular}

Fuente: Elaboración propia. 
En relación a la facilidad del modelo en la práctica profesional, el $75 \%$ del total de profesionales lo consideran fácil, frente a un poco más del $5 \%$ que piensa que es de aplicación dificultosa. Son los técnicos en educación social los que valoran como más fácil de aplicar la metodología, seguidos muy de cerca de los trabajadores sociales. En lo que se refiere al lugar de trabajo, aunque los profesionales de servicios sociales opinan mayoritariamente que es fácil de desarrollar y aplicar, las restantes categorías de respuesta han obtenido porcentajes dignos de tomar en cuenta: un $16 \%$ considera que su facilidad es media, y un $8 \%$, difícil. Los profesionales de dependencia y de atención a personas sin hogar han ofrecido valoraciones menos dispersas, pero en ambos casos son positivas y valoran la metodología como de fácil aplicación.

\subsection{Ventajas metodológicas y factores de dificultad}

La Tabla 2 muestra los resultados ofrecidos por los profesionales acerca de las ventajas que ofrece la metodología en la práctica profesional.

Como se puede observar en los porcentajes de respuesta, los profesionales encuestados consideran que el modelo ofrece ventajas importantes. Prácticamente todos los participantes consideran que mejora la intervención profesional, y seis de cada diez, que ofrece nuevos instrumentos y recursos de planificación. Igual de importante es el cambio en el papel del usuario en el proceso de intervención social: menos de la mitad de los profesionales consideran que mediante la metodología se producen cambios en la organización, o que supone un cambio de principios y objetivos.
Son los profesionales de la educación social los que la han considerado más ventajosa, pues más de la mitad de los participantes han considerado que aporta mejoras en cada uno de los ítems analizados. Para todos ellos, las mejoras en la intervención son la ventaja más evidente, seguida -con el mismo nivel de importancia- de la aportación de nuevos conocimientos, de las mejoras organizativas, de las mejoras en los instrumentos de intervención y de un cambio en el papel del usuario. Seis de cada diez profesionales consideran que el modelo supone un cambio en los principios de la intervención social. Para los trabajadores sociales, además de las mejoras en la intervención como principal valoración, la mejora en los instrumentos de planificación es la segunda ventaja metodológica. La mitad de los profesionales piensan que produce un cambio en el papel del usuario. A diferencia de los educadores sociales, para menos del $50 \%$ el resto de ítems no suponen ventajas importantes.

La totalidad de los profesionales de servicios sociales valoran como principales ventajas la mejora en la intervención profesional y en los instrumentos utilizados para planificar. Para el resto de variables, supone una ventaja en opinión de aproximadamente la mitad de los encuestados. Los profesionales de dependencia/asistencia domiciliaria también valoran como principal ventaja la mejora en la intervención: esta vez, la mitad de los encuestados lo considera así. Las opiniones del resto de ítems no son importantes. Todos los profesionales de atención a personas sin hogar consideran como principal ventaja de la metodología la mejora en la intervención, seguida por la mejora en los instrumentos utilizados y, en tercer lugar, la introducción de nuevos conocimientos, el cambio del papel del usuario y los cambios en la organización.

Tabla 2. Opinión de los profesionales sobre las ventajas de la planificación centrada en la persona (\%)

\begin{tabular}{|l|c|c|c|c|c|c|}
\hline & Conocimientos & Principios & Organización & Intervención & Instrumentos & Usuario \\
\hline Total & 55,6 & 47,2 & 47,2 & 94,4 & 66,7 & 61,1 \\
\hline Trabajador social & 43,8 & 37,5 & 31,3 & 93,8 & 68,8 & 50,0 \\
\hline Educador social & 66,7 & 60,0 & 66,7 & 100,0 & 66,7 & 66,7 \\
\hline Trabajador familiar & 100,0 & 50,0 & 0,0 & 50,0 & 50,0 & 50,0 \\
\hline Servicios sociales & 56,0 & 52,0 & 48,0 & 100,0 & 68,0 & 56,0 \\
\hline Dependencia & 25,0 & 25,0 & 0,0 & 50,0 & 25,0 & 75,0 \\
\hline $\begin{array}{l}\text { Atención a personas sin } \\
\text { hogar }\end{array}$ & 66,7 & 33,3 & 66,7 & 100,0 & 83,3 & 66,7 \\
\hline
\end{tabular}

Fuente: Elaboración propia. 
Tabla 3. Opinión de los profesionales sobre los inconvenientes de la planificación centrada en la persona (\%)

\begin{tabular}{|c|c|c|c|c|c|c|c|}
\hline & Conocimientos & Organización & Recursos & Interés & Utilidad & $\begin{array}{c}\text { No } \\
\text { aplicabilidad }\end{array}$ & Carga trabajo \\
\hline Total & 33,3 & 55,6 & 16,7 & 0,0 & 5,6 & 0,0 & 19,4 \\
\hline $\begin{array}{l}\text { Trabajador } \\
\text { social }\end{array}$ & 18,8 & 18,8 & 12,5 & 0,0 & 12,5 & 0,0 & 12,5 \\
\hline $\begin{array}{l}\text { Educador } \\
\text { social }\end{array}$ & 40,0 & 40,0 & 20,0 & 0,0 & 0,0 & 0,0 & 26,7 \\
\hline $\begin{array}{l}\text { Trabajador } \\
\text { familiar }\end{array}$ & 50,0 & 50,0 & 50,0 & 0,0 & 0,0 & 0,0 & 50,0 \\
\hline $\begin{array}{l}\text { Servicios } \\
\text { sociales }\end{array}$ & 24,0 & 56,0 & 20,0 & 0,0 & 8,0 & 0,0 & 24,0 \\
\hline Dependencia & 50,0 & 75,0 & 25,0 & 0,0 & 0,0 & 0,0 & 0,0 \\
\hline $\begin{array}{l}\text { Atención a } \\
\text { personas sin } \\
\text { hogar }\end{array}$ & 50,0 & 50,0 & 0,0 & 0,0 & 0,0 & 0,0 & 16,7 \\
\hline
\end{tabular}

Fuente: Elaboración propia.

En la Tabla 3 se recogen las respuestas relativas a las posibles limitaciones que puede suponer en su implementación.

Según las valoraciones de la mitad de los profesionales, el principal inconveniente de la metodología son las dificultades organizativas del servicio en que trabajan. El resto de factores son considerados como limitaciones por menos de la mitad de los participantes. Concretamente, el segundo motivo de dificultad sería la falta de conocimientos relativos a la práctica metodológica, y el tercer y cuarto lugar corresponden, respetivamente, al aumento de carga de trabajo y a la falta de recursos disponibles, valorados como inconvenientes por menos del $20 \%$ del total de profesionales. El último motivo de dificultad es la falta de utilidad práctica del modelo. Ningún profesional ha percibido como inconveniente la falta de interés o la no aplicabilidad metodológica.

Según el perfil profesional, son los trabajadores familiares los que valoran como mayores las dificultades: la mitad de ellos consideran que la falta de conocimiento sobre la metodología, las dificultades organizativas, la falta de recursos y el aumento en la carga de trabajo son limitaciones de la práctica metodológica. Cuatro de cada diez educadores sociales consideran que la falta de conocimientos y las dificultades en la organización del servicio son los hándicaps más importantes, seguidos del aumento en la carga de trabajo y la falta de recursos. Las valoraciones de los trabajadores sociales no superan en ningún caso el $20 \%$ del total. Las dificultades más relevantes serían la falta de conocimientos metodológicos y las dificultades organizativas, seguidas por la falta de recursos, el aumento en la carga de trabajo y la falta de utilidad de la metodología en la práctica profesional, siendo los trabajadores sociales los únicos que han considerado importante esta limitación.

Si se analizan las valoraciones según el lugar de trabajo, son los profesionales de servicios sociales los que encuentran más limitaciones en la metodología. Las dificultades organizativas suponen un obstáculo para la mitad de ellos, mientras que la falta de conocimientos sobre la metodología, la falta de recursos para llevarla a la práctica y el aumento en la carga de trabajo son hándicaps para menos del $25 \%$ de los profesionales. Los trabajadores de la dependencia o la asistencia domiciliaria valoran las dificultades organizativas como principal inconveniente, seguidas de la falta de conocimientos y de recursos. Los profesionales de atención a personas sin hogar consideran como limitaciones más importantes la falta de conocimientos de la práctica metodológica y las dificultades organizativas, ambas valoradas por la mitad de los trabajadores. El otro inconveniente sería el aumento de la carga de trabajo, considerado por el $16,7 \%$ de profesionales.

\section{Discusión}

Son numerosos los estudios que analizan la opinión de los usuarios de servicios sociales (Medina Tornero, 1996; Medina Tornero y Medina Ruiz, 2011; De la Peña, 2014; Arenas Martínez, 2015), la calidad de los servicios (Sacanell, 1995; Sánchez Aguado, 2011) o incluso el síndrome de desgaste profesional (burnout) [Barrera, Malagón y Sarasola, 2015], siendo más escasos los que recogen las opiniones de los profesionales de servicios sociales acerca de su labor cotidiana.

Entre estos últimos, las investigaciones valoran la importancia de la perspectiva profesional en la intervención social con diferentes y muy variados objetivos, que se mueven entre la valoración de aspectos generales de los servicios sociales o las funciones profesionales (Federación Sartu, 2010; Arenas Martínez, 2011; Cortinas, 2012; Alsinet et al., 2013; Rodríguez Álvarez, 2015) y las evaluaciones de proyectos concretos de intervención (Elósegui et al., 2014).

El objetivo principal de la presente investigación ha sido averiguar qué opinan los profesionales de 
servicios sociales acerca de la planificación centrada en la persona. Los resultados principales del estudio realizado sobre 36 profesionales ofrecen información importante acerca de la aplicabilidad, complejidad y facilidad de la metodología en la intervención social, así como de cuáles son los inconvenientes a la hora de adaptarla y también sus ventajas. A partir de estas informaciones, se puede valorar la posibilidad de aplicar el paradigma en los servicios sociales, al tener en cuenta la opinión de quienes diariamente implementan acciones sociales que pretenden mejorar la calidad de vida de las personas, objetivo fundamental del modelo.

La valoración realizada no deja lugar a dudas ni respecto a la aplicabilidad de la metodología, ni respecto a su facilidad de implementación, siendo los profesionales de la educación social quienes la consideran más aplicable, por encima de las valoraciones que los trabajadores sociales y familiares realizan. Fantova (2004) recoge la planificación centrada en la persona como uno de los modelos de gestión de calidad más importantes en el ámbito de la intervención social, basado en el concepto de calidad de vida y la centralidad de la persona. Lima (2012) asegura que uno de los retos más importantes del trabajo social es desarrollar garantías metodológicas que incluyan acciones que tengan como eje principal a la persona, hecho que parecen testimoniar los participantes en el presente estudio con sus valoraciones.

Ante cualquier tipo de cambio, suelen aparecer miedos y temores entre las personas que se encargan de su desarrollo. Las preguntas acerca de las ventajas e inconvenientes de la metodología en el ámbito de los servicios sociales pretendían conocer estos miedos, pero también las potencialidades de los profesionales: los primeros, para incorporarlos en el proceso de adaptación del modelo; y las segundas, para potenciarlas en su implementación.

Más de la mitad de los profesionales han valorado como un elemento negativo los cambios organizativos que el modelo produciría al adaptarla en los servicios sociales. No se trata de un porcentaje elevado, pero sí suficientemente importante como para que cobre importancia. Estos cambios organizacionales no suceden únicamente en niveles o planos superiores de gestión; también lo hacen en la práctica cotidiana, en la organización de los equipos de trabajo. En esta línea, Arenas Martínez (2011), en un estudio cualitativo y de análisis institucional, tras recoger y examinar las opiniones de los trabajadores y educadores sociales de atención primaria, concluyó que un requisito importante para que los servicios sociales se adaptaran a las nuevas realidades actuales era que las metodologías aplicables se implementaran en organizaciones capaces de responder con agilidad a las necesidades de las personas. Para Duque (2012), es preciso que se produzca un cambio de actitud de responsables políticos, directivos y profesionales para pasar de un modelo de recursos a uno de atención personalizada. Por tanto, las dudas de los participantes en el estudio acerca de los cambios que pueden producirse en la organización deben tenerse muy en cuenta.

Una tercera parte de los participantes valoran la falta de conocimientos acerca de la metodología como segundo motivo de preocupación. Entre las muy escasas investigaciones que analizan la opinión de los profesionales acerca de la planificación, Coyle y Moloney (1999), al estudiar las resistencias iniciales al cambio de paradigma de intervención social, concluyeron que la necesidad de formar a los equipos de trabajo en la metodología era fundamental para vencer los miedos y las limitaciones al cambio. Los profesionales participantes en ese estudio añadieron que era necesaria la formación continua si se pretendía implementar con éxito la metodología. En un ejercicio de revisión de la literatura acerca las políticas y de las prácticas del modelo desarrolladas en Inglaterra, Dowling et al. (2006) encontraron que uno de los factores que determinaba la implementación más lenta o parcial de una nueva metodología era la falta de formación de los profesionales. Los resultados del presente estudio son similares a los obtenidos por Arellano y Peralta (2016) cuando analizaron las dificultades de la aplicación del paradigma por parte de profesionales del ámbito de la discapacidad, autoras para quienes la falta de conocimientos y formación era un problema importante.

Las dos últimas limitaciones que los participantes en la presente investigación consideran relevantes son el aumento de la carga de trabajo que puede suponer la adaptación de la planificación centrada en la persona en los servicios sociales y la falta de recursos necesarios para su aplicación. En ambos casos, no superan el $20 \%$ de las respuestas obtenidas, pero deben tomarse en cuenta. La percepción profesional acerca de la carga de trabajo se ha considerado en estudios como el de Hayajneh y Shehadeh (2014), quienes analizaron el impacto de un enfoque centrado en la persona en la carga de trabajo de los profesionales de atención directa de personas con alzhéimer residentes en centros.

Al observar las respuestas obtenidas en relación a las ventajas metodológicas, los resultados muestran valoraciones muy positivas por parte de los profesionales. Prácticamente el $95 \%$ considera que mejoraría la intervención profesional, hecho realmente destacable, y para dos terceras partes ofrece nuevos instrumentos y recursos de planificación. Igual de importante es el cambio en el papel del usuario en el proceso de intervención social. Los resultados se asemejan a los que ofrecen algunos de los estudios empíricos sobre el impacto del modelo (Holburn et al., 2004; Robertson et al., 2006; Peterson, Buchanan y Falkmer, 2014), al afirmar que la metodología mejora la calidad de vida de los destinatarios de la intervención, así como las relaciones entre los equipos profesionales, y entre éstos y las personas usuarias, además de promover el papel de la persona en su cambio vital. 
Menos de la mitad de los profesionales consideran que mediante la metodología se producen cambios en la organización, o que supone un cambio de principios y objetivos. Es preciso destacar esta conclusión, ya que se relaciona con las expuestas por numerosos autores (Holburn y Vietze, 1999; Flannery et al., 2000; Merkel-Holguin, 2004), quienes afirman que los cambios organizacionales son una de las más importantes limitaciones para el éxito en la implantación del modelo. Para los profesionales participantes en el presente estudio, los cambios en la organización no suponen una ventaja evidente, y esto puede tener una doble lectura: por una parte, puede significar que no consideren importante que la planificación implique una modificación en el funcionamiento de la organización donde desarrollan su labor profesional; pero también podría traducirse como que la organización ya participa de algunos de sus principios y, por ese motivo, las transformaciones no son tan evidentes.

El presente estudio pretende ser un primer acercamiento a la opinión que los profesionales de servicios sociales realizan sobre la posible aplicación de la planificación centrada en la persona en la intervención social, y como tal, adolece de varias limitaciones. La más importante es el tamaño de la muestra, que reduce en gran medida la generalización de resultados. En todo caso, la presencia en la muestra de profesionales de diferentes disciplinas hace que esta dificultad se vea reducida en cuanto a la representación de los diferentes profesionales que forman parte de los equipos de trabajo a quienes va dirigida la investigación. Igualmente, es necesario tener en cuenta que la participación voluntaria de los profesionales encuestados también supone un hándicap, ya que puede existir una predisposición a valorar positivamente los elementos que se les propone evaluar.

Sin embargo y pese a estas dificultades, se puede afirmar que el presente estudio es una primera toma de contacto acerca de las valoraciones que profesionales de servicios sociales realizan sobre las posibilidades de la aplicación de la metodología en este ámbito. Siendo un paradigma cuyos principios y fundamentos respetan los derechos y libertades de las personas y promueven sus decisiones, es importante seguir fomentando investigaciones que aceleren su implementación en los servicios sociales. La formación y la sensibilización de profesionales y gestores es imprescindible para conseguirlo. 
AGUIRRE, B. (2013): “El acompañamiento social personalizado como fórmula innovadora de respuesta a personas con trastorno mental y en exclusión social", Zerbitzuan, n- 54 , págs. 127-137 [<https://doi.org/10.5569/11347147.54.08>].

ALSINET, C. et al. (2013): Impacte en les famílies de Lleida dels canvis en la renda minima d'inserció, Barcelona, Col-legi Oficial de Treball Social de Catalunya.

ARELLANO, A.; y PERALTA, F. (2016): “La planificación centrada en la persona: un ejemplo de buena práctica en el ámbito de la discapacidad intelectual", Contextos Educativos, ํㅜ 19, págs. 195-212 [<http://revistas.ces.edu.co/ index.php/psicologia/article/view/2891/>].

ARENAS MARTíNEZ, M. (2015): “Calidad percibida en los servicios sociales de inclusión. Una aproximación desde el caso de Avilés (Asturias)", Cuadernos de Trabajo Social, vol. 28, no 1 , págs. 115-125.

- (2011): “Crítica y razón de los servicios sociales de base. Atención y proactividad en el Estado social", Zerbitzuan, no 49, págs. 39-53 [<https://doi. org/10.5569/1134-7147.49.04>].

ARIAS MARTÍNEZ, B. et al. (2010): "Evaluación de la calidad de vida en personas drogodependientes mediante el modelo de Rasch", Revista Española de Drogodependencias, vol. 35, ํㅜ 2, págs. 206-219.

BARRERA, E.; MALAGÓN, J. L.; y SARASOLA, J. L. (2015): "Trabajo social, su contexto profesional y el Síndrome de Burnout”, Comunitania. Revista Internacional de Trabajo Social y Ciencias Sociales, $\mathrm{n}-9$, págs. 51-71 [<http://www. comunitania.com/evaristo-barrera-jose-luis- malagon-jose-luis-sarasola-trabajo-socialsu-contexto-profesional-y-el-sindrome-deburnout/>]

BENITO, E.; ALSINET, C.; y MACIÁ, A. (2015): “La calidad de vida en personas sin hogar con y sin discapacidad. El caso de la ciudad de Lleida" (comunicación), IX Jornadas Científicas Internacionales de Investigación sobre Personas con Discapacidad. 18-20 de marzo de 2015, Salamanca.

BOERSMA, P. et al. (2014): "The art of successful implementation of psychosocial interventions in residential dementia care: A systematic review of the literature based on the RE-AIM framework", International Psychogeriatrics, vol. $27, \mathrm{n}{ }^{0}$ 1, págs. 19-35.

BRONFENBRENNER, U. (1979): The Ecology of Human Development: Experiments by Nature and Design, Cambridge, Harvard University Press.

COGHLAM, D. (1993): "A person-centred approach to dealing with resistance to change", Leadership \& Organization Development Journal, vol. 14, $\mathrm{n}$ 은, págs. 10-14.

CORTINAS, J. (2012): “La identidad profesional de los trabajadores sociales como elemento clave en el acceso a los programas de rentas mínimas: el caso de Catalunya", Zerbitzuan, ํㅜ 51, págs. 95-105 [shttps://doi.org/10.5569/11347147/51.06)].

COYLE, K.; y MOLONEY, K. (1999): “The introduction of person-centred planning in an Irish agency for people with intellectual disabilities: An introductory study", Journal of Vocational Rehabilitation, vol. 12, n- 3, págs. 175-180.

DE LA PEÑA, E. (2014): “Análisis de la satisfacción y de la calidad percibida por las personas atendidas 
en los servicios sociales comunitarios del Centro Municipal de Servicios Sociales Delicias del Ayuntamiento de Zaragoza", Cuadernos de Trabajo Social, vol. 27, no 1, págs. 115-125.

DOWLING, S. et al. (2006): Person-centred Planning in Social Care: A Scoping Review, York, York Publishing Services.

DUQUE, J. M. (2012): “Redescubrimiento de los servicios sociales de atención primaria: hacia un (nuevo) modelo de atención personal y comunitaria". Zerbitzuan, no 0 52, págs. $23-44$ [shttps://doi. org/10.5569/1134-7147.52.02>].

EDWARSSON, D.; SANDMAN, P. O.; y BORELL, L. (2014): "Implementing national guidelines for personcentered care of people with dementia in residential aged care: Effects on perceived person-centeredness staff strain, and stress of conscience", International Psychogeriatrics, vol. $26, \mathrm{n}^{0} \mathrm{7}$, págs. 1.171-1.179.

ELÓSEGUI, E. et al. (2014): “Elección consensuada de una herramienta común de valoración sociosanitaria en la CAPV", Zerbitzuan, nํ 56 , págs. 41-57 [<https://doi.org/10.5569/11347147.53.02>].

FANTOVA, F. (2004): "Gestión de calidad en los sistemas de servicios sociales de las comunidades autónomas: perspectivas generales y propuestas de avance", Boletín del CIES, n- 21 .

FEDERACIÓN SARTU (2010): "Tendencias sociales e inclusión social”, Zerbitzuan, no 48, págs. 137-144 [<http://www.zerbitzuan.net/documentos/ zerbitzuan/Tendencias \%2osociales $\% 20$ e $\% 20$ inclusion \%20social.pdf>].

FERNÁNDEZ MUÑOZ, J. N. (2011): "Servicios sociales, derechos de ciudadanía y atención centrada en la persona", TS Nova. Trabajo Social y Servicios Sociales, $\mathrm{n}{ }^{0} 3$, págs. $37-48$.

FLANNERY, B. K. et al. (2000): "Person centered assessment and planning: An introduction to new tools", Journal for Vocational Special Needs Education, vol. $23, \mathrm{n}$ - 3 , págs. $15-20$.

HAYAJNEH, F. A.; y SHEHADEH, A. (2014): “The impact of adopting person-centred care approach for people with Alzheimer's on professional caregivers' burden: An interventional study", International Journal of Nursing Practice, vol. 20, no- 4, págs. 438-445.

HOLBURN, S. (2003): “Cómo puede la ciencia evaluar y mejorar la planificación centrada en la persona”, Siglo Cero, vol. 34, no- 4, págs. 48-64.

HOLBURN, S.; y VIETZE, P. (1999): “Acknowledging barriers in adopting person-centered planning", Mental Retardation, vol 37, nํㅡ 2, págs. 117-124.

HOLBURN, S. et al. (2004): “The Willowbrook Futures project: A longitudinal analysis of personcentered planning", American Journal on Mental Retardation, № 109, págs. 63-76.

LIMA, A. I. (2012): "Trabajo social, nuevos contextos y nuevos compromisos", Azarbe. Revista Internacional de Trabajo Social y Bienestar, $\mathrm{n}$ 을 1 , págs. $73-86$.

MARTÍNEZ RODRÍGUEZ, T. (2013): "La atención centrada en la persona. Algunas claves para avanzar en los servicios gerontológicos", Actas de la Dependencia, no 8, págs. 25-47.
MARTÍNEZ RODRÍGUEZ, T. et al. (2014): La atención centrada en la persona. ¿Cómo llevarla a la práctica?, serie Modelo de Atención Centrada en la Persona, no 2, Departamento de Empleo y Políticas Sociales del Gobierno Vasco y Matia Instituto [<http://www.matiainstituto.net/es/ publicaciones/cuadernos-practicos-modelode-atencion-centrado-en-la-persona-2-comollevarla-la〉].

MEDINA TORNERO, M. E. (1996): "Evaluación de la calidad asistencial en Servicios Sociales", Psychosocial Intervention, vol. $5, \mathrm{n}^{0} \mathbf{0}$, págs. 23-42.

MEDINA TORNERO, M. E.; y MEDINA RUIZ, E. (2011): “Análisis de la calidad percibida en usuarios/as de servicios sociales", Zerbitzuan, ํㅜ 50, págs. 85-100 ['https://doi.org/10.5569/11347147.50.07)].

MERKEL-HOLGUIN, L. (2004): "Sharing power with the people: Family group conferencing as a democratic experiment", Journal of Sociology and Social Welfare, vol. 31, nํㅜ 1, págs. 155-173.

MOUNT, B.; y ZWERNIK, K. (1988): It's Never Too Early. It's Never Too late. A Booklet about Personal Futures Planning, Minnesota, Metropolitan Council.

NEILL, M. et al. (2009): "A positive approach to risk requires person-centred thinking", Tizard Learning Disability Review, vol. 14, no 4, págs. 17-24.

PALLISERA, M. (2011): “La planificación centrada en la persona (PCP): una vía para la construcción de proyectos personalizados con personas con discapacidad intelectual", Revista Iberoamericana de Educación, vol. 56, nํㅜ 3 .

PEARPOINT, J.; O'BRIEN, J.; y FOREST, M. (1993): Path: A Workbook for Planning Possible Positive Futures: Planning Alternative Tomorrows with Hope for Schools, Organizations, Businesses, Families, Toronto, Inclusion Press.

PETERSON, S.; BUCHANAN, A.; y FALKMER, T. (2014): “The impact of services that offer individualised funds, shared management, person-centred relationships, and self-direction on the lived experiences of consumers with mental illness", International Journal of Mental Health Systems, vol. 8, no 20 , págs. 1-14 [khttp://www.ncbi.nlm. nih.gov/pmc/articles/PMC4061914/>].

ROBERTSON, J. et al. (2006): "Longitudinal analysis of the impact and cost of person-centered planning for people with intellectual disabilities in England", American Journal on Mental Retardation, vol. 111, nํ․ 4, págs. 400-416.

RODRÍGUEZ ÁLVAREZ, Maㅡ D. (2015): "Redes profesionales e interdisciplinariedad en trabajo social comunitario: una respuesta integral a problemas sociales multidimensionales", Zerbitzuan, nํㅗ 58, págs. 41-50 [rhttps://doi. org/10.5569/1134-7147.58.04)].

SACANELL, E. (1995): "El concepto de calidad en la gestión de los servicios sociales", Zerbitzuan, no 20, págs. 63-88 [<http://www.zerbitzuan. net/documentos/zerbitzuan/Calidad $\% 20$ en $\% 20$ la \%2ogestion \%20de \%2olos \%20 servicios \%20sociales.pdf〉].

SÁNCHEZ AGUADO, A. (2011): "La calidad en la gestión de los Servicios Sociales Municipales. Algunas 
experiencias de INTRESS", TS Nova. Trabajo Social y Servicios Sociales, $\mathrm{n}$ ㅇ 3, págs. 49-54.

SCHALOCK, R. (1996): “The quality of life: It's definition and measurement", Research in Developmental Disabilities, vol. 16, no 1, págs. 51-74.

- (1995): Quality of Life: Its Conceptualization, Measurement and Use, Washington D.C., American Association of Mental Retardation.

SCHALOCK, R.; y VERDUGO, M. A. (2009): “Revisión actualizada del concepto de calidad de vida", en VERDUGO, M. A. (coord.), Cómo mejorar la calidad de vida de las personas con discapacidad: instrumentos y estrategias de evaluación, Salamanca, Amarú Ediciones.
- (2003): Calidad de vida. Manual para profesionales de la educación, salud y servicios sociales, Madrid, Alianza Editorial.

SMULL, M. W. (1998): “After the plan”, en O’BRIEN, J.; y O'BRIEN, C. L. (eds.), A Little Book about Person Centered Planning, Toronto, Inclusion Press.

VERDUGO, M. A. (dir.) (2008): Formulari de l'Escala Gencat de Qualitat de vida. Manual d'aplicació de l'Escala Gencat de Qualitat de Vida, Generalitat de Catalunya, Departament d'Acció Social i Ciutadania.

VERDUGO, M. A. et al. (2010): Impacto social del programa ECA Caja Madrid de empleo con apoyo, Salamanca, Instituto Universitario de Integración en la Comunidad. 